\title{
Disruption of attention to novel events after frontal lobe injury in humans
}

Kirk R Daffner, M Marsel Mesulam, Philip J Holcomb, Vivian Calvo, Diler Acar, Alexandra Chabrerie, Ron Kikinis, Ferenc A Jolesz, Dorene M Rentz, Leonard F M Scinto

Brigham Behavioral Neurology Group, Laboratory of Higher Cortical Functions, Division of Cognitive and Behavioral Neurology, Brigham and Women's Hospital, Harvard Medical School, 221 Longwood Avenue, Boston, MA 02115, USA

K R Daffner

V Calvo

D Acar

D M Rentz

L F M Scinto

Center for Behavioral and Cognitive Neurology,

Northwestern University Medical School, Chicago, IL 60611, USA

M M Mesulam

Department of Psychology, Tufts University, Medford, MA 02155, USA

P J Holcomb

MRI Division, Surgical Planning Laboratory, Department of Radiology, Brigham and Women's Hospital, Harvard Medical

School, Boston, MA

02115, USA

A Chabrerie

R Kikinis

F A Jolesz

Correspondence to: Dr Kirk R Daffner, Division of Cognitive and Behavioral Neurology, Brigham and Women's Hospital, Harvard Medical School, 221 Longwood Avenue, Boston, MA 02115, USA email

krdaffner@bics.bwh.harvard.ed

Received 8 April 1999 and in revised form

6 July 1999

Accepted 16 July 1999

\section{Abstract}

Objective-To investigate whether frontal lobe damage in humans disrupts the natural tendency to preferentially attend to novel visual events in the environment.

Methods-Nine patients with chronic infarctions in the dorsolateral prefrontal cortex (DLPFC) and 23 matched normal controls participated in a study in which subjects viewed repetitive background stimuli, infrequent target stimuli, and novel visual stimuli (for example, fragmented or "impossible" objects). Subjects controlled viewing duration by a button press that led to the onset of the next stimulus. They also responded to targets by pressing a foot pedal. The amount of time spent looking at the different kinds of stimuli, and the target detection accuracy and speed served as dependent variables.

Results-Overall, normal controls spent significantly more time than frontal lobe patients looking at novel stimuli. Analysis of responses across blocks showed that initially frontal lobe patients behaved like normal controls by directing more attention to novel than background stimuli. However, they quickly began to distribute their viewing time evenly between novel and background stimuli, a pattern that was strikingly different from normal controls. By contrast, there were no differences between frontal lobe patients and normal controls for viewing duration devoted to background and target stimuli, target detection accuracy, or reaction time to targets. Frontal lobe patients did not differ from normal controls in terms of age, education, estimated IQ, or mood, but were more apathetic as measured by self report and informants' judgments. Attenuated responses to novel stimuli significantly correlated with degree of apathy.

Conclusions-This study demonstrates that DLPFC injury selectively impairs the natural tendency to seek stimulation from novel and unusual stimuli. These data provide the first quantitative behavioural demonstration that the human frontal lobes play a critical part in directing and sustaining attention to novel events. The impairment of novelty seeking behaviour may contribute to the characteristic apathy found in patients with frontal lobe injury. (F Neurol Neurosurg Psychiatry 2000;68:18-24)

Keywords: frontal lobes; attention; novelty; apathy
Although animal studies have strongly suggested that frontal lobe injury often leads to diminished attention to novel events and reduced exploratory behaviours, ${ }^{1-8}$ there has been very limited research on this phenomenon in humans. ${ }^{9-13}$ In the current study, the duration of viewing time that a subject voluntarily chose to devote to different kinds of stimuli was measured to quantify novelty seeking behaviour. ${ }^{14-22}$ Our goal was to determine whether damage to the frontal lobes reduces the natural tendency to allocate more attention to novel stimuli. Such disruption may contribute to the symptoms of apathy and disengagement often noted in patients with frontal lobe injury. ${ }^{23-27}$

\section{Subjects and methods}

SUBJECTS

Brain CT and MRI of patients discharged from the Brigham and Women's Hospital with a diagnosis of stroke were reviewed. Patients whose infarctions were located in the dorsolateral part of the frontal lobes (with no extension into the parietal cortex) were recruited. Patients with a history of previous strokes, extensive white matter changes, alcohol misuse, or dementia were excluded. Eight patients underwent research MRI, with three dimensional reconstruction of images. ${ }^{28}$ One patient had research CT because a pacemaker made her ineligible for MRI.

Lesion localisation was based on the Damasio template system. ${ }^{29}$ In patients who were able to have MRI, infarct localisation was confirmed by reviewing the three dimensional images that were reconstructed from the MRI data $\operatorname{set}^{28}$ after the boundaries of the lesion had been traced on each slice. Six patients had right frontal infarctions; two had left. One patient had had small, bilateral frontal infarctions. The infarctions in all patients were centred in the dorsolateral prefrontal cortex. Mean duration poststroke was 1.7 years (SD 0.8), fig 1 . Normal controls $(n=23)$ were recruited through advertisements in the Boston community. Subjects were excluded if they had a history of cerebrovascular disease, alcohol misuse, dementia, or a focal neurological examination. Informed consent was obtained from all subjects.

Subjects completed the American version of the National adult reading test ${ }^{3031}$ and the Raven coloured progressive matrices test ${ }^{32}$ to determine an estimated IQ score. In normal controls, the two test scores were averaged, whereas in frontal lobe patients the higher of the two test scores was used to try to obtain an 


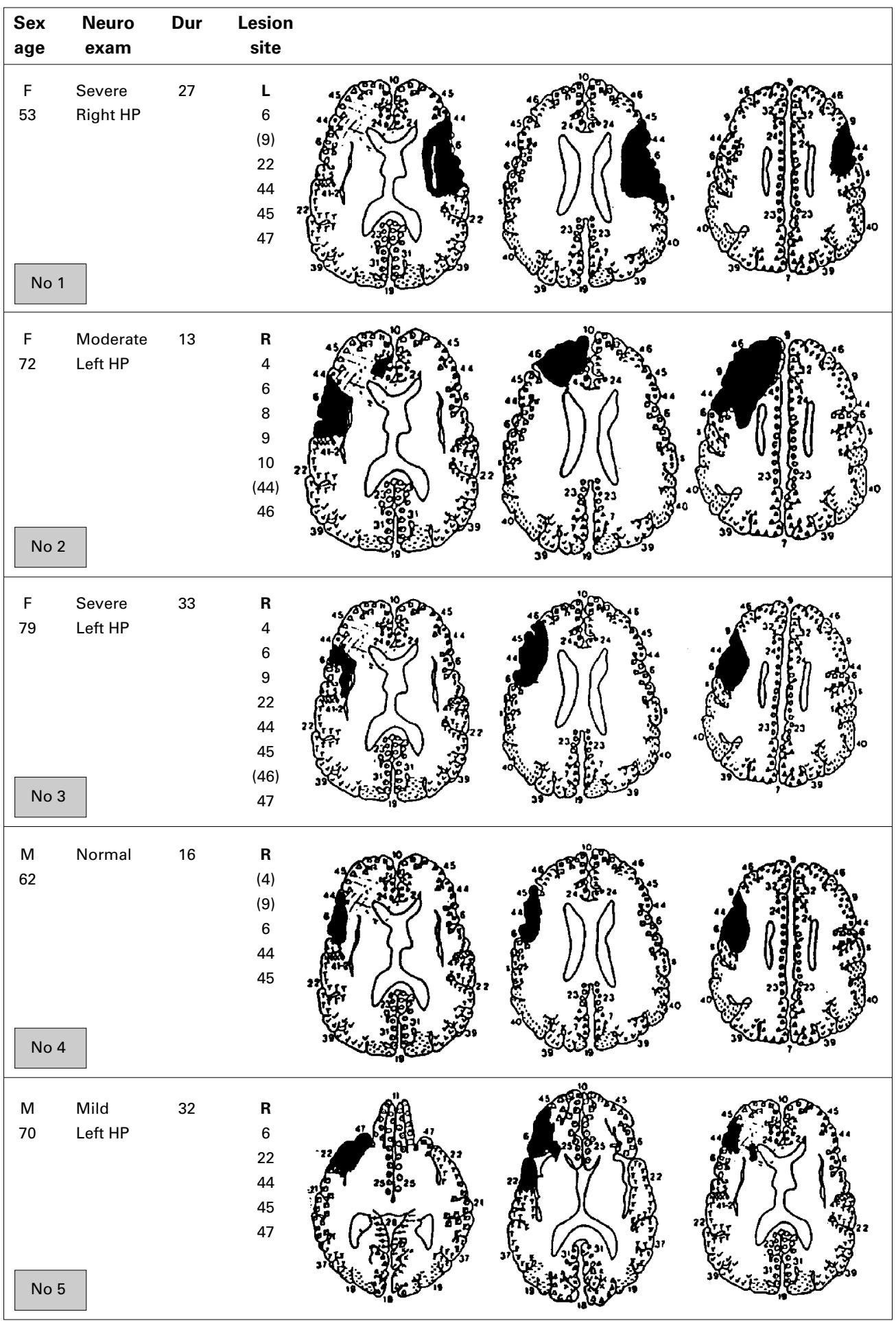

Figure 1 Summary of patient information. Boundaries of the infarct are shown on representative Damasio ${ }^{29}$ templates. Patients $1-8$ are based on MRI. Patient 9 is based on CT. Lesion site=Hemisphere and Brodmann areas; $R=$ right; $L=l e f t ;$ numbers in () indicate that the infarct is just touching the specified area; Dur=duration in months; HP=hemiparesis.

accurate estimate of their premorbid intellectual capacities. Subjects were also evaluated neuropsychologically with the mini mental state examination (MMSE), ${ }^{33}$ digit span subtest from the WAIS-R, ${ }^{34}$ CERAD word list recall and recognition test, ${ }^{35}$ Benton visual retention test (BVRT), ${ }^{36}$ and the comprehension and naming subtests of the multilingual aphasia examination (MAE).$^{37}$ Subjects completed the apathy scale ${ }^{38} 39$ (a 14 item question- naire that inquires about a subject's level of interest, motivation, and concern) and the Zung depression scale ${ }^{40}$ (a 20 item survey about the subject's mood and affective state). Informants who knew the subjects well completed a personality and behavioural inventory developed in our laboratory ${ }^{41}$ that included four items evaluating the subject's degree of apathy by assessing his or her level of initiation, participation, interest, and motivation. 


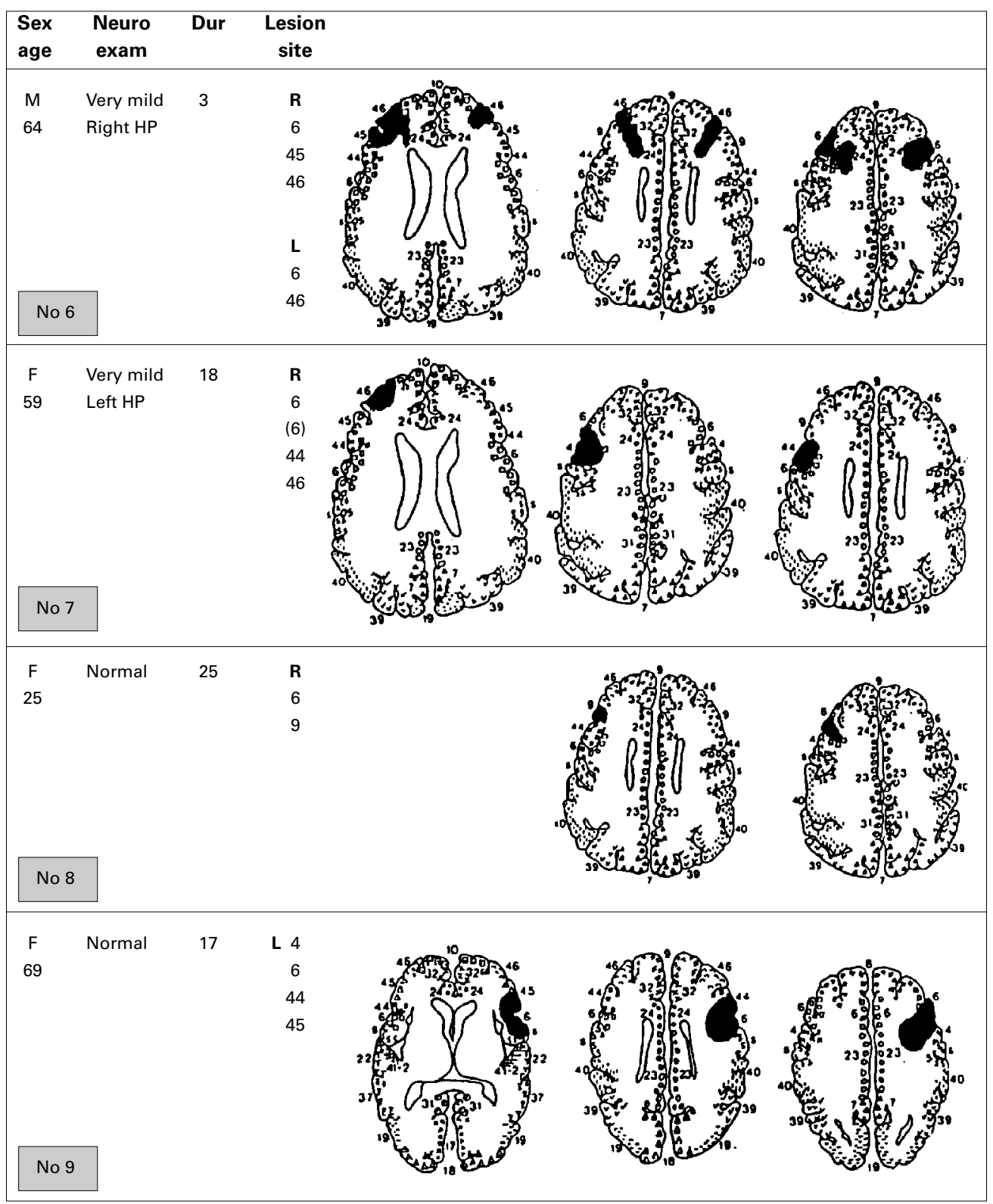

Figure 1 Continued

EXPERIMENTAL PROCEDURES

Three hundred line drawings were presented at the centre of a computer monitor. There were six blocks of 50 stimuli each, with brief breaks between each block. All stimuli subtended a visual angle of about $2.75^{\circ}$ along their longest dimension. In each block, three categories of visual stimuli were presented in random order: (1) A repetitive background stimulus (a triangle) $-70 \%$ frequency, (2) target stimulus (upside down triangle)-15\% frequency, and (3) novel stimuli, randomly drawn from a set of unusual/unfamiliar line drawings (for example, fragmented or "impossible" objects) shown only one time each-15\% frequency (fig 2). Many of the novel stimuli came from the collection of drawings that have been used by Kosslyn $e t a l^{42}$ and Kroll and Potter. ${ }^{43}$

Subjects were told that the study was investigating how people look at different kinds of line drawings. They were informed that they would be viewing a set of drawings and that they could look at each picture for however long they liked. They controlled the viewing duration by a button press that triggered the onset of the next stimulus. Subjects were explicitly told that they would not be asked questions about the pictures at the conclusion of the experiment. They also were asked to respond to a designated "target" stimulus by pressing a foot pedal. We called the targets "sequence markers" and indicated that their main purpose was to help the experimenters keep track of where they were in the sequence of drawings presented. For the stroke patients, the responding hand and foot used were ipsilateral to the lesion site. For the normal controls and the patient with bilateral lesions, the responding hand and foot were randomly assigned.

Data were analysed using repeated measures analysis of variance (ANOVA) (with GeisserGreenhouse corrections ${ }^{44}$ or Student's $t$ tests. Data sets that were not normally distributed were transformed (for example, inverse 


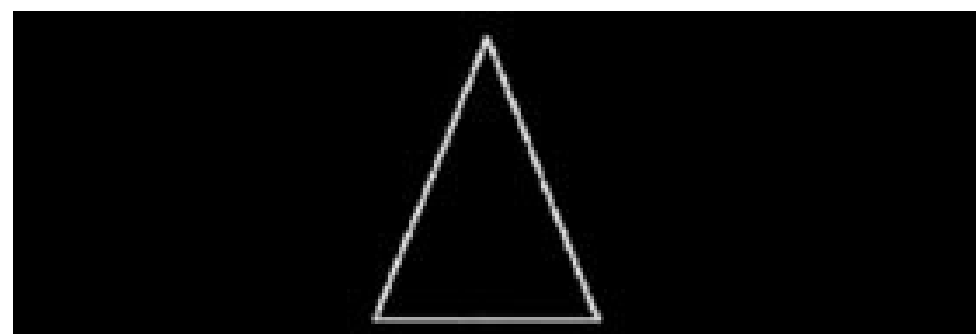

Background

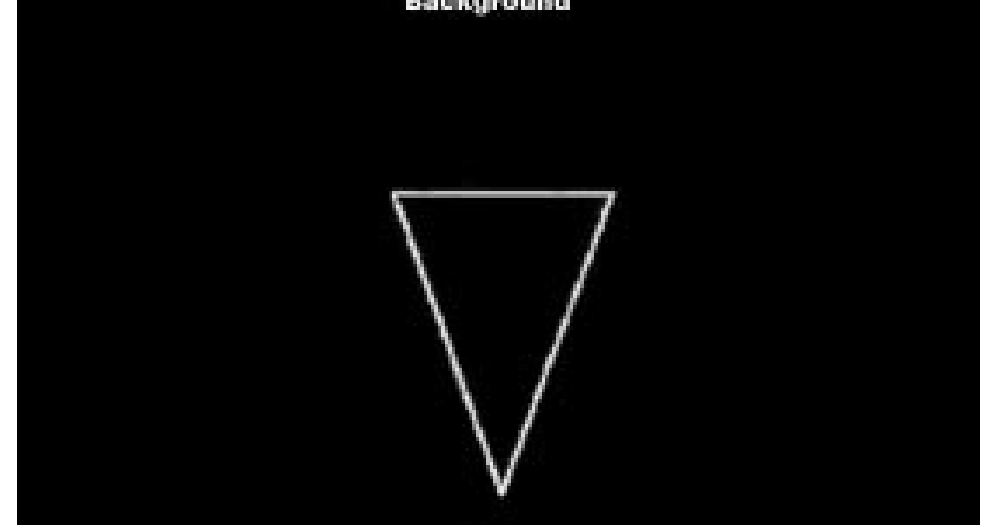

Target

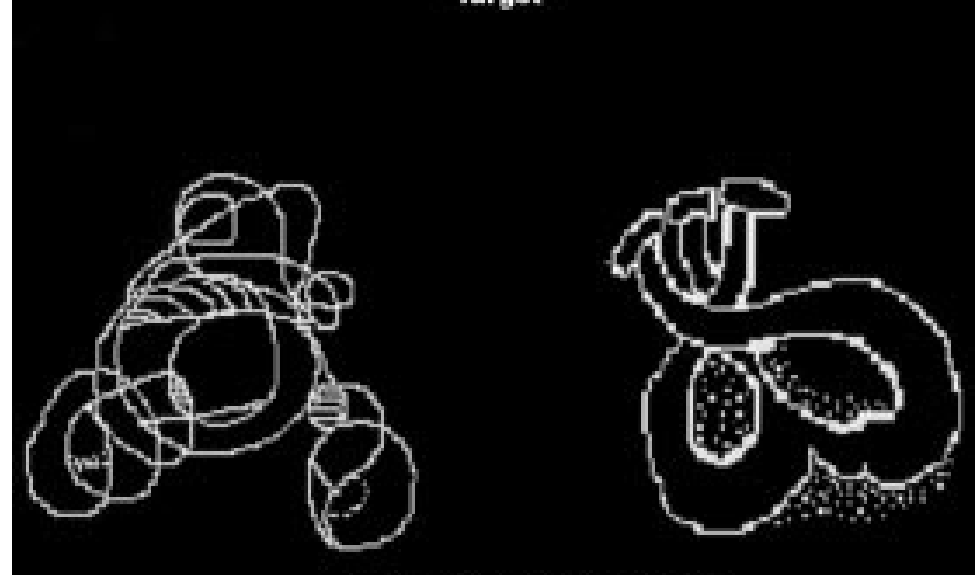

2 Examples of novel stimuli

Figure 2 Repetitive background stimulus (70\% frequency), target stimulus (15\% frequency), and two examples of the novel stimuli (15\% frequency).

function) before statistical analyses. If assumptions for parametric analyses were violated, Mann-Whitney $U$ tests were employed. Correlational analysis was accomplished using Spearman's $r$. All $\mathrm{p}$ values reported are two tailed.

Results

SUBJECT CHARACTERISTICS

As shown in table 1, patients with frontal lobe strokes and normal controls did not differ

Table 1 Subject characteristics

\begin{tabular}{lccl}
\hline & $\begin{array}{c}\text { Normal controls } \\
(n=23) \text { mean (SD) }\end{array}$ & $\begin{array}{c}\text { Frontal lobe patients } \\
(n=9) \text { mean }(S D)\end{array}$ & $p$ \\
\hline Age & $68.3(7.2)$ & $61.2(16.3)$ & $\mathrm{NS}$ \\
Education (y) & $16.1(3.0)$ & $15.4(2.5)$ & $\mathrm{NS}$ \\
Estimated IQ & $121.5(5.5)$ & $116.4(10.0)$ & $\mathrm{NS}$ \\
Zung score (20-80) & $30.8(5.1)$ & $37.0(11.7)$ & $\mathrm{NS}$ \\
$\begin{array}{l}\text { Apathy scores } \\
\text { - Self report scale (0-42) }\end{array}$ & $5.9(3.7)$ & $11.3(6.5)$ & $\mathrm{p}<0.04$ \\
- Informant based inventory (0-40) & $6.1(5.0)$ & $17.0(6.4)$ & $\mathrm{p}<0.00005$ \\
\hline
\end{tabular}

For both Zung and apathy scales, higher numbers indicate more severe symptomatology.

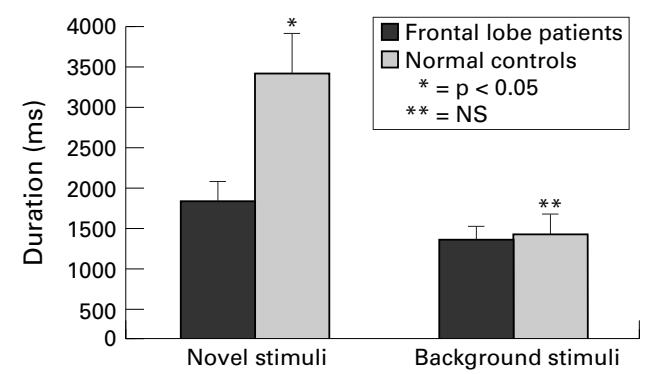

Figure 3 Viewing duration on novel and background stimuli (mean (SEM) in ms) for normal controls and frontal lobe patients. There was a significant stimulus type by group interaction $(p<0.009)$. Normal controls spent significantly more time than frontal lobe patients looking at novel stimuli $(p<0.05)$; however, the two groups did not differ in their viewing duration on background stimuli.

significantly in terms of age, education, estimated IQ, ${ }^{30-32}$ or Zung depression scale scores. ${ }^{40}$ The mean Zung scores for both groups were below the cut off for clinically significant depression. There were no group differences in performance on $\mathrm{MMSE},{ }^{33}$ digit span, ${ }^{34} \mathrm{CERAD}$ word recall and recognition test, ${ }^{35}$ or the naming subtest of the MAE. ${ }^{37}$ Frontal lobe patients performed worse than normal controls on the BVRT $^{36} \quad(\mathrm{p}<0.02)$ and the comprehension subtest of the $\mathrm{MAE}^{37}(\mathrm{p}<0.002)$. Frontal lobe patients exhibited increased apathy as measured by self-report on the apathy scale ${ }^{3839}(\mathrm{p}<0.04)$ and informant based judgments on the personality and behavioural inventory ${ }^{41}(p<0.00005)$. According to these measures, the apathy exhibited by the frontal lobe patients was in the mild range of severity.

\section{EXPERIMENTAL DATA}

Overall viewing durations on background and novel stimuli differed across the groups (significant group by stimulus type interaction, $\mathrm{p}<0.009)$. Although normal controls spent more time than frontal lobe patients looking at novel stimuli $(\mathrm{p}<0.05)$, there were no group differences in viewing duration on background stimuli ( $p>0.6$, fig 3 ).

Subjects varied in terms of the rate at which they tended to move through the stimulus set. Response times in stroke subjects may have been affected by altered speed of motor or cognitive processing. To help control for these factors, we constructed a measure of proportionality (viewing duration novels/viewing duration backgrounds) for each subject. Compared with frontal lobe patients, normal controls looked significantly longer at the novel stimuli relative to the background stimuli (frontal lobe patients: 1.37 (SEM 0.11) v normal controls: 2.68 (SEM 0.37), p<0.004).

The values (viewing duration novel/ background) derived for subjects inversely correlated with their degree of apathy as assessed by informants (Spearman's $r=-0.56, \mathrm{p}<0.002$ ) and self report (Spearman's $r=-0.41, \mathrm{p}<0.03$ ). By contrast, there was no correlation between apathy scores and reaction time to targets, \% correct hits, or viewing duration on targets.

Analysis of the data across the six blocks showed a significant stimulus type by block by group interaction ( $\mathrm{p}<0.008$, fig 4$)$. In block 1 , 

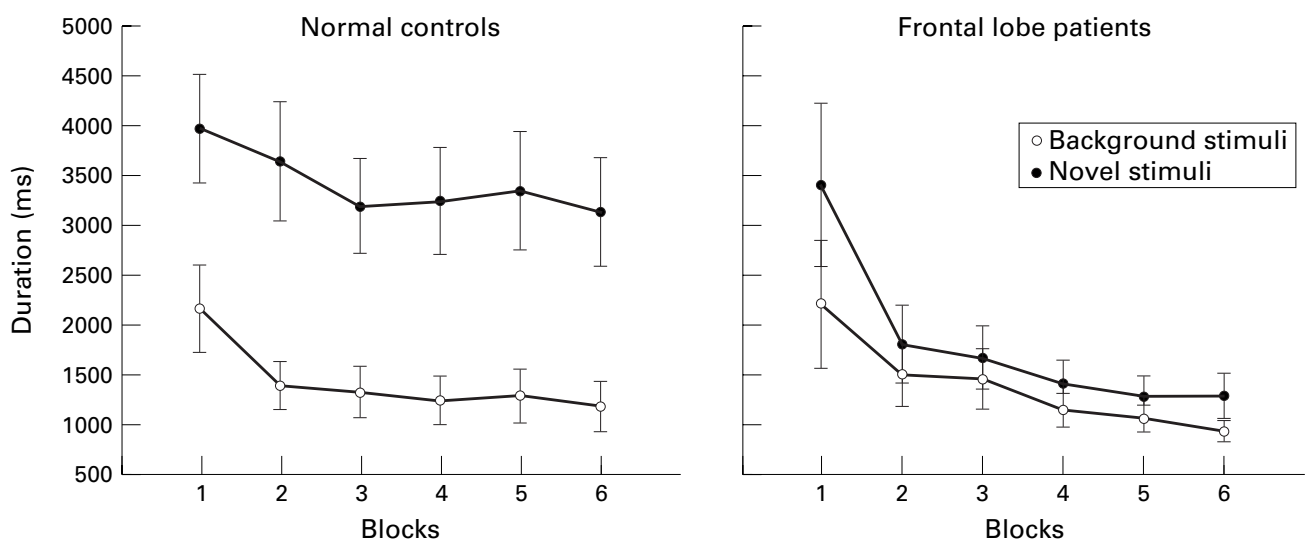

Figure 4 Viewing duration across blocks on novel and background stimuli (mean (SEM) in ms) for normal controls and frontal lobe patients. There was a significant stimulus by block by group interaction $(p<0.008)$. Unlike normal controls, frontal lobe patients demonstrate a rapid decline in viewing duration on novel stimuli across blocks.

there were no differences in response to background and novel stimuli between normal controls and frontal lobe patients ( $\mathrm{p}>0.9)$. Both groups spent more time looking at novel than background stimuli (main effect of stimulus type, $\mathrm{p}<0.00005$; no stimulus type by group interaction). Frontal lobe patients exhibited a rapid decline in dwell time on novel stimuli across blocks $(p<0.0002)$, with an almost $50 \%$ reduction between blocks 1 and $2(\mathrm{p}<0.02)$. In blocks $2-6$, frontal lobe patients did not look significantly longer at novel than background stimuli (no main effect of stimulus type; no block by stimulus type interaction). By contrast, normal controls did not show a decline in viewing time on novel stimuli across blocks $1-6(p>0.2)$ and continued to attend much longer to novel than background stimuli in blocks 2-6 $(\mathrm{p}<0.00005)$. Both groups exhibited a similar pattern of habituation across blocks in response to the repetitive background stimuli (main effect of blocks, $\mathrm{p}<0.00005$; no block by group interaction.)

In terms of responses to target stimuli, there were no significant group differences in reaction time, $\%$ correct hits, number of false alarms, viewing duration on target stimuli, or the ratio of viewing duration on target stimuli/ viewing duration on background stimuli (table 2 ). Both frontal lobe patients and normal controls spent more time viewing target than background stimuli $(\mathrm{p}<0.00005$; no stimulus type by group interaction). This pattern was maintained throughout all blocks (no main effect of blocks). For both groups, the viewing duration on targets diminished across blocks $(\mathrm{p}<0.00005)$ and the reaction time in response to targets got faster across blocks $(\mathrm{p}<0.00005)$. However, the effect was similar for both groups

Table 2 Responses to target stimuli

\begin{tabular}{llll}
\hline & $\begin{array}{l}\text { Normal controls mean } \\
(S D)\end{array}$ & $\begin{array}{l}\text { Frontal lobe patients } \\
\text { mean (SD) }\end{array}$ & $p$ \\
\hline Reaction time (ms) & $1342(684)$ & $1338(547)$ & NS \\
\% Hits & $0.97(0.05)$ & $0.93(0.13)$ & NS \\
False alarms & $0.45(0.45)$ & $2.3(5.9)$ & NS \\
Viewing duration (ms) & $2612(1040)$ & $2897(1354)$ & NS \\
Viewing duration targets & $2.2(0.6)$ & $2.3(0.9)$ & NS \\
\hline Viewing duration backgrounds & & & \\
\hline
\end{tabular}

(no block by group interactions). Also, the \% hits on targets did not differ across blocks for either group.

To what extent did the size of the lesion play a part in the outcome? Some patients had relatively circumscribed lesions within the dorsolateral prefrontal cortex, whereas others had large lesions that involved much more of the frontal lobes. We compared the three patients with the largest lesions (patients 1, 2, 3) with the three patients who had the smallest ones (patients 7, 8, 9). Given the small sample size, non-parametric statistics were employed. The ratio of the viewing duration novels/viewing duration backgrounds was almost identical for the two subgroups (small lesion group: 1.30 (SEM 0.16), large lesion group: 1.22 (SEM $0.10), p>0.4)$. There were also no group differences in degree of apathy. However, the patients with larger lesions exhibited a greater disruption of activities of daily living ${ }^{45}$ $(p<0.05)$. In addition, the two patients in our sample with left sided infarctions (patients 1,9) were compared with two patients with right sided infarctions of similar size and distribution (patients 3,4). The ratio of viewing duration novels/viewing duration backgrounds, and degree of apathy and depression were very similar between the left and right sided groups, with no statistical differences noted.

\section{Conclusion}

Animal studies have shown that damage to the frontal cortex impairs orienting responses to novel stimuli. ${ }^{1-8}$ Lesioned animals do not tend to exhibit physiological and behavioural responses that differentiate novel from repetitive stimuli. $^{1-6}$ Moreover, frontal lobe damage in animals disrupts a range of exploratory activities and blunts their innate novelty seeking behaviour. ${ }^{52527}$ In humans, it is commonly seen that injury to the frontal lobes, especially when large and bilateral, can lead to disengagement and apathy. ${ }^{23-25} 27$ Few studies have examined the potential cognitive substrate of this phenomenon.

Our study shows that damage to the human frontal lobes disrupts the preferential allocation of attention to novel stimuli as measured by the 
duration of voluntary viewing times. Compared with normal controls, frontal lobe patients spent less time looking at novel relative to background stimuli. This effect cannot be attributed to differences in age, education, premorbid IQ, or depression. Several other explanations for the reduced viewing time of frontal lobe patients on novel stimuli need to be considered, including: (1) a different interpretation than normal controls of the purpose of the study; (2) an inability to identify novel stimuli; (3) a non-specific reduction of attentional capacity; and (4) a selective disruption of attention to novel events.

Despite task instructions, normal controls may have "anticipated" a subsequent test of their memory and thus looked at novel items longer to encode them better. A failure of frontal lobe patients to generate such an anticipatory response could have led them to look at novel stimuli for shorter durations than normal controls. However, this explanation seems inadequate. All subjects were "debriefed" immediately after participation in the experiment and none of the normal controls indicated that they examined the novel stimuli longer out of a concern for the possibility of being tested subsequently on them. More importantly, this explanation cannot account for the fact that frontal lobe patients initially display a clear preference for the novel figures. This finding also strongly suggests that frontal lobe patients are able to identify stimuli that are novel as well as control subjects.

A generalised inability of frontal lobe patients to sustain attention to the content of stimuli could lead to rhythmically advancing the display without consideration of the figures being viewed. Several findings argue against this hypothesis. Frontal lobe patients were able to sustain attention to target stimuli throughout the task. Like normal controls, their accuracy in response to targets was maintained and their reaction time improved across blocks. Furthermore, frontal lobe patients did not distribute their viewing times evenly across all stimulus types, but spent significantly more time looking at targets.

Damage to the frontal lobes seems to lead to a selective inability to sustain interest in novelty. The overall viewing duration and habituation of response to background stimuli across blocks did not differ between frontal lobe patients and normal controls. Also, frontal lobe patients and normal controls had a very similar pattern of responses to target stimuli (reaction times and viewing durations) overall and across blocks. Taken together these findings make it implausible that the decreased viewing time allocated to novel stimuli exhibited by frontal lobe patients is explained by a non-specific impairment of attentional capacity or arousal over the course of the experiment. Rather, the disruption of attention seen in patients with frontal lobe injury is relatively specific to stimuli that are novel.

The infarcts in all of the patients involved the dorsolateral prefrontal cortex (Brodmann areas $9,44,45,46$, or 47). Patients who had small strokes in this region exhibited as severe a dis- ruption of novelty seeking behaviour as patients with lesions involving much wider areas. This finding suggests that the dorsolateral prefrontal cortex may make a particularly important contribution to responses to novelty, as has been shown in primate studies. ${ }^{25}$

Apathy, as defined by diminished goal directed behaviour related to reduced motivation, initiative, and curiosity, ${ }^{47}$ is a common outcome of frontal lobe damage. ${ }^{23-26}{ }^{46}$ Consistent with the literature, the frontal lobe patients in this study were more apathetic than normal controls (as measured by self report and informants). It is likely that many factors contribute to the development of apathy. The failure to devote appropriate attentional resources to novel events may undermine a critical early step in more complex aspects of exploratory behaviour. Marin $e t a l^{48}$ found that the degree of apathy in neurological patients correlated with reduced levels of spontaneous exploratory and novelty seeking behaviour in an experimental setting that was measured according to specific research criteria. In the current study, the strong correlation between apathy and quantitatively assessed reduction in novelty seeking behaviour strengthens the contention that the two phenomena are related.

The underlying physiology that supports the role of the frontal lobes in attention to novel events remains to be determined. Knight and colleagues $^{1149-52}$ have shown that patients with damage to the dorsolateral prefrontal cortex exhibit a substantially reduced "novelty" P3 wave, a frontally distributed electrophysiological marker of orienting responses to novel events. ${ }^{11} 2253-55$ We have shown that in normal subjects, the amplitude of the novelty P3 in frontal regions strongly predicts the duration of subsequent viewing directed to novel stimuli. ${ }^{22}$ The frontally generated P3 may reflect the activity of a process that dynamically links attentional resources to novel events. Further work is needed to define the specific contributions of the frontal lobes to novelty seeking behaviour. For example, although several brain regions, including the hippocampus, facilitate orienting to novelty, ${ }^{51525657}$ the dorsolateral prefrontal cortex may be critical in sustaining interest in novelty over time. The approach reported here will allow researchers to pursue such hypotheses experimentally. This initial study provides the first quantitative behavioural demonstration that the human frontal lobes play an important part in directing and sustaining attention to novel events. Injury to frontal regions markedly disrupts these exploratory activities, which likely contributes to the apathy noted clinically.

We thank Dr B Kennedy for his statistical advice. We appreciate Dr S M Kosslyn and Drs J F Kroll and M C Potter for allowing us to use their stimulus sets. We acknowledge Barbara Vericker and Lise Bliss for their expert secretarial and administrative assistance. This work was supported by NIH grant K20MH01378, Brigham behavioral Neurology Group, Laboratory of Higher Cortical Functions, Division of Cognitive and behavioral Neurology, Brigham and Women's Hospital, Harvard Medical School, Boston, MA 02115, USA.

1 Butter CM. Habituation of responses to novel stimuli in monkeys with selective frontal lesions. Science 1964;144:313-15. 
2 French GM, Harlow HF. Locomotor reaction decrement in normal and brain-damaged rhesus monkeys. ₹ Comp normal and brain-damaged rhe

3 Glaser EM, Griffin JP. Influence of the cerebral cortex on habituation. F Physiol 1962;160:429-45.

4 Hagamen WD, Lance EM, Ungewitter LH. Increased responsiveness to stimuli following lesions of the forebrain. Anat Rec 1959;133:387-8.

5 Jacobsen CF. Studies of cerebral function in primates I: the function of the frontal association areas in monkeys. Comp Psychol Monogr 1936;13:3-50.

6 Kimble DP, Bagshaw MH, Pribram KH. The GSR of monkeys during orienting and habituation after selective partial ablations of the cingulate and frontal cortex. Neuropsychologia 1965;3:121-8.

7 Morgan CT, Wood WM. Cortical localization of symbolic processes in the rat. II. Effect of cortical lesions upon delayed alternation in the rat. $\mathcal{F}$ Neurophysiol 1943;6:17380 .

8 Welker WI. An analysis of exploratory and play behavior in animals, in Fiske DW, Maddi SR, eds. Functions of varied experience. Homewood, IL: Dorsey Press, 1961:175-226.

9 Hutton JT, Albrecht JW, Shapiro I, et al. Visual information processing and dementia. Neuroophthalmology 1987;7:10512.

10 Hutton JT, Johnston CW, Shapiro I, et al. Oculomotor programming disturbances in the dementia syndrome. Percept Mot Skills 1979;49:312-14.

11 Knight RT. Decreased response to novel stimuli after prefrontal lesions in man. Electroencephalogr Clin Neurophysiol 1984;59:9-20.

12 Luria AR. The frontal lobes and the regulation of behavior. In: Pribram KH, Luria AR, eds. Psychophysiology of the frontal lobes. New York, NY: Academic Press, 1973:3-26.

13 Luria AR, Karpov BA, Yarbuss AL. Disturbances of active visual perception with lesions of the frontal lobes. Cortex 1966;2:202-12.

14 Berlyne D. Conflict, arousal and curiosity. New York, NY: McGraw-Hill, 1960

15 Daffner KR, Scinto LFM, Weintraub S, et al. Diminished curiosity in patients with probable Alzheimer's disease as
measured by exploratory eye movements. Neurology measured by

16 Daffner KR, Scinto LFM, Weintraub S, et al. The impact of aging on curiosity as measured by exploratory eye movements. Arch Neurol 1994;51:368-76.

17 Humphrey NK. Interest and pleasure: two determinants of a monkey's visual preferences. Perception 1972;1:395-416.

18 Loftus GR, Mackworth NH. Cognitive determinants of fixation location during picture viewing. F Exp Psychol Hum Percept Perform 1978;4:565-72.

19 Mackworth NH, Morandi AJ. The gaze selects informative details within pictures. Percept Psychophys 1967;2:547-52.

20 Noton D, Stark L. Eye movements and visual perception. Sci Am 1971;224:34-43.

21 Vurpillot E. The development of scanning strategies and their relation to visual differentiation. 7 Exp Child Psychol 1968;6:632-50.

22 Daffner KR, Mesulam MM, Scinto LFM, et al. Regulation of attention to novel stimuli by frontal lobes: an event-related potential study. Neuroreport 1998;9:787-91.

23 Damasio AR, Anderson SW. The frontal lobes. In: Heilman KM, Valenstein E, eds. Clinical neuropsychology. New York, NY: Oxford University Press, 1993:409-60.

24 Fuster JM. The prefrontal cortex: anatomy, physiology, and neuropsychology of the frontal lobe. New York, NY: Raven Press, 1989.

25 Mesulam MM. Frontal cortex and behavior. Ann Neurol 1986;19:320-5

26 Stuss DT, Benson DF. The frontal lobes, New York, NY: Raven Press, 1986.

27 Mesulam MM. From sensation to cognition. Brain 1998; 121:1013-52.

28 Shenton ME, Kikinis R, Jolesz FA, et al. Abnormalities of the left temporal lobe and thought disorder in schizophrenia: a quantitative magnetic resonance imaging schizophrenia: a quantitative magnetic
study. N Engl f Med 1992;327:604-12.

29 Damasio H, Damasio AR. Lesion localization in neuropsychology. New York, NY: Oxford University Press, 1989.

30 Nelson HE, O'Connell A. Dementia: the estimation of premorbid intelligence levels using the new adult reading test. Cortex 1978;14:234-44.

31 Ryan J, Paolo A. A screening procedure for estimating premorbid intelligence in the elderly. Clin Neuropsycho 1992;6:53-62.
32 Raven JC, Court JH, Raven J. Coloured progressive matrices, section 2. In: Manual for Raven's progressive matrices and vocabulary scales. Oxford: Oxford Psychologists Press, 1995:1-73.

33 Folstein MF, Folstein SE, McHugh PR. Mini-mental state. 7 Psychiatr Res 1975;12:189-98.

34 Wechsler D. Wechsler adult intelligence scale: revised manual. New York, NY: The Psychological Corporation, 1981.

35 Morris JC, Heyman A, Mohs RC, et al. The consortium to establish a registry for Alzheimer's disease (CERAD). Part
I. Clinical and neuropsychological assessment of Alzheimer's disease. Neurology 1989;39:1159-65

36 Benton AL. Revised visual retention test: clinical and experimental applications. New York: The Psychological Corporation, 1963.

37 Benton AL, DeS Hamsher K. Multilingual aphasia examination. Manual of instructions. Iowa City, IA: AJA Associates, 1983

38 Starkstein SE, Fedoroff JP, Price TR, et al. Apathy following cerebrovascular lesions. Stroke 1993;24:1625-30.

39 Starkstein SE, Migliorelli R, Manes F, et al. The prevalence and clinical correlates of apathy and irritability in Alzheimer's disease. Eur f Neurol 1995;2:540-6.

40 Zung WWK. A self rating depression scale. Arch Gen Psychiatry 1965;12:63-70.

41 Daffner KR, Mesulam MM, Cohen LG, et al. Mechanisms underlying diminished novelty-seeking behavior in patients with probable Alzheimer's disease. Neuropsychiatry Neuropsychol Behav Neurol 1999;12:58-66.

42 Kosslyn SM, Alpert NM, Thompson WL, et al. Identifying objects seen from different viewpoints: a PET investigation. Brain 1994;117:1055-71.

43 Kroll JF, Potter MC. Recognizing words, pictures, and concepts: a comparison of lexical, object and reality decisions. Fournal of Verbal Learning and Verbal Behaviour 1984;23:39-66.

44 Geisser S, Greenhouse S. On methods in the analysis of profile data. Psychometrika 1959;24:95-112.

45 Weintraub $\mathrm{S}$. The record of independent living: an informant completed measure of activities of daily living and behavior in elderly patients with cognitive impairment. $A m$ 7 Alzheimer's Care 1986;1:35-9.

46 Cummings JL. Behavioral disorders associated with frontal lobe injury. In: Clinical neuropsychiatry. Orlando, FL: Grune and Stratton, 1985:57-67.

47 Marin RS. Differential diagnosis and classification of apathy. Am f Psychiatry 1990;147:22-30.

48 Marin RS, Biedrzycki RC, Firinciogullari S. Reliability and validity of the apathy evaluation scale. Psychiatry Res 1991; 38:143-62.

49 Knight RT. Evoked potential studies of attention capacity in human frontal lobe lesions. In: Levin HS, Eisenberg HM, Benton AL, eds. Frontal lobe function and dysfunction. Oxford: Oxford University Press, 1991:139-53.

50 Knight RT. Distributed cortical network for visual attention. f Cogn Neurosci 1997;9:75-91.

51 Knight RT, Scabini D. Anatomic bases of event-related potentials and their relationship to novelty detection in humans. F Clin Neurophysiol 1998;15:3-13.

52 Knight RT, Nakada T. Cortico-limbic circuits and novelty: a review of EEG and blood flow data. Rev Neurosci 1998;9:57-70

53 Courchesne E, Hillyard SA, Galambos R. Stimulus novelty, task relevance and the visual evoked potential in man. Electroencephalogr Clin Neurophysiol 1975;39:131-43.

54 Hillyard SA, Picton TW. Electrophysiology of cognition. In: Plum F, Mountcastle VB, Geiger ST, eds. The handbook of physiology. Section I. The nervous system. Vol V. Higher functions of the brain part 2. Bethesda, MD: American Physiological Society, 1987:519-84.

55 Baudena P, Halgren E, Heit G, et al. Intracerebral potentials to rare target and distractor auditory and visual stimuli. III. Frontal cortex. Electroencephalogr Clin Neurophysiol 1995; 94:251-64.

56 Halgren E, Baudena P, Clarke JM, et al. Intracerebral potentials to rare target and distractor auditory and visual potentials to rare target and distractor auditory and visual Electroencephalogr Clin Neurophysiol 1995;94:229-50.

57 Knight RT. Contribution of human hippocampal region to novelty detection. Nature 1996;383:256-9. 